\title{
Relational Quantum Mechanics and the Determinacy Problem*
}

\author{
Matthew Brown
}

July 9, 2006

This paper is a DRAFT.

You may cite it as such, but beware its draftiness.

Please do not quote directly.

Comments are welcome and encouraged.

email: mattbrown@ucsd.edu

web: http://thehangedman.com/philosophy/

\begin{abstract}
Quantum mechanics describes certain systems as being in superpositions of their properties, yet every measurement on every system that we are able to perform yields a determinate result. The "orthodox" formulation of the theory builds this in by including a postulate that such superpositions "collapse" at the time of measurement. This strategy fails to really explain why such measurements are determinate, is unacceptably imprecise, and makes observation basic in fundamental physical theory, which looks like the wrong sort of process for the job. The problem of the interpretation of quantum mechanics is the problem of finding a more satisfactory understanding of the formalism in the face of these problems. Carlo Rovelli's relational interpretation of quantum mechanics holds that a system's states or the values of its
\end{abstract}

*This project began in an independent study with Craig Callender, who provided much help in terms of direction, discussion, comments, and continuing support. Thanks also to Jeffrey Barrett for reading and commenting on an earlier draft of this paper, including helping me find some clearer and more economical way of putting some crucial points. 
physical quantites as normally conceived only exist relative to a cut between a system and an observer or measuring instrument. Furthermore, on Rovelli's account, the appearance of determinate observations from pure quantum superpositions happens only relative to the interaction of the system and observer. Jeffrey Barrett has pointed out that certain relational interpretations suffer from what we might call the "determinacy problem," but the interpretations that Barrett considers make facts relative to branches of the universal wave function rather than to system/observer cuts. Thus, Barrett misclassifies Rovelli's interpretation, which differs from the interpretations that Barrett explicitly worries about and has the resources to escape his particular criticisms. Rovelli's interpretation still leaves us with a paradox having to do with the determinacy of measurement outcomes, which can be accepted only if we are willing to give up on certain elements of the "absolute" view of the world.

\section{Introduction}

Quantum mechanics describes certain systems as being in superpositions of their properties, yet every measurement on every system that we are able to perform yields a determinate result. The "orthodox" formulation of the theory builds this in by including a postulate that such superpositions "collapse" at the time of measurement. This strategy fails to really explain why such measurements are determinate, is unacceptably imprecise, and makes observation basic in fundamental physical theory, which looks like the wrong sort of process for the job. The problem of the interpretation of quantum mechanics is the problem of finding a more satisfactory understanding of the formalism in the face of these problems.

Carlo Rovelli's [Rov96, Laud05] relational interpretation of quantum mechanics holds that a system's states ${ }^{1}$ or the values of its physical quantites as normally conceived only exist relative to a cut between system and observer

\footnotetext{
${ }^{1}$ I speak of the "state" of the system throughout the paper, though, in the considered view of both the supporters of the relational interpretation [Laud05] and myself, it is infelicitous to call the wavefunction / Hilbert-space vector of the system "the state" or to treat it as what corresponds to the classical state. Better to speak of the quantities of physical variables or operators like "position" and "momentum" as those things that have reality (relatively speaking). Nevertheless, as nothing crucial is lost in the translation for the purposes of this paper, I speak of states in order to stick closer to Rovelli's original presentation [Rov96] and achieve maximal familiarity.
} 
(alternatively: measurement system, exosystem, metasystem, measurement frame). Furthermore, on Rovelli's account, the appearance of (absolutely) determinate observations from pure (superposed) quantum states happens only relative to the interaction of the system with the exosystem. Jeffrey Barrett [Bar99] has pointed out that certain relational interpretations like Rovelli's suffer from what we might call the "determinacy problem," but the interpretations that Barrett considers make facts relative to branches of the universal wave function rather than to cuts. While Rovelli's interpretation differs from the sorts of interpretations the Barrett explicitly worries about and has the resources to escape his particular criticisms, the relational interpretation still results in a paradox. The problem manifests itself for Rovelli's interpretation not as a problem of determinate experience, as Barrett worries about, and it doesn't arise for the same reason it arises in interpretations of Everett or on Mermin's interpretation, but it instead manifests as our inability to give any absolute or gods-eye-view descriptions of events.

First, I will give a brief description of the view, following Rovelli's presentation. Next, I will discuss Barrett's criticisms of David Mermin's interpretation, and then contrast Rovelli's interpretation with Mermin's in a way that draws out further features of Rovelli's interpretation as well as showing how it escapes Barrett's criticisms. In the next section, I discuss a further puzzle for Rovelli's interpretation, and I finish by considering two strategies for dissolving the puzzle that the relationalist can employ (the second better than the first).

\section{Relational Quantum Mechanics}

Rovelli [Rov96] takes his cues on the interpretation of quantum mechanics from Einstein, ${ }^{2}$ particularly Einstein's landmark 1905 paper on special relativity. Einstein accomplishes two things in his paper on special relativity that inspire Rovelli. First, Einstein provides an interpretation of an already existing formalism (the Lorentz transformations), answering the charges that the formalism is unreasonable or inconsistent by criticizing an implicit assumption (absolute simultaneity) that is "inappropriate to describe reality when velocities are high"[Rov96, p. 2]. Second, Einstein does not merely tack an interpretation on to the formalism, but he attempts to understand

\footnotetext{
${ }^{2} \mathrm{He}$ is not the first to have done so. Both Heisenberg and Bohr saw themselves as making moves analogous to Einstein's relativity. See also [Fink96, Fink04].
} 
or in some sense derive the formalism on the basis of some simple physical principles (ones that may seem contradictory given the inappropriate assumptions: in the case of special relativity, "equivalence of inertial observers and universality of the speed of light"[Rov96, p. 2]).

Rovelli is interested in following Einstein's strategy in both respects when giving his interpretation of quantum mechanics. His program would start with some simple physical assertions, showing that they entail a rejection of some inappropriate assumption(s), and then derive the formalism of quantum theory from them. He admits that this project is not yet completely successful, and so splits the discussion into two parts: a motivation of some basic ideas about quantum mechanics from a discussion of the "third person problem," the conjunction of which lead us to the discovery of the inappropriate assumptions that form our uneasy attitude towards quantum mechanics, and an attempt to reconstruct the formalism (somewhat informally) from a small number of postulates suggested by the first discussion, dealing with the information systems have about each other. ${ }^{3}$ I will focus on the first part of Rovelli's discussion.

Rovelli draws his main conclusions from a discussion of the "third man problem," which we can see as follows: ${ }^{4}$ Suppose observer (or measuring apparatus) $O$ is measuring a property of system $S$ (e.g., a Stern-Gerlach apparatus measuring the x-spin of an electron, with possible values of spinup and spin-down). We'll represent the two eigenstates of that property as $|\uparrow\rangle_{S}$ and $|\downarrow\rangle_{S}$. If $S$ is in the arbitrary state $|\psi\rangle_{S}=\alpha|\uparrow\rangle_{S}+\beta|\downarrow\rangle_{S}$, when $O$ measures the electron's x-spin, he will find the value 'up' (with probability $\left|\alpha^{2}\right|$ ) or 'down' (with probability $\left|\beta^{2}\right|$ ). Now, suppose that $O$ measures $S$ and in fact finds it to be up. We usually represent this with the following state transition:

$$
\begin{aligned}
t_{1} & \rightarrow t_{2} \\
\alpha|\uparrow\rangle_{S}+\beta|\downarrow\rangle_{S} & \rightarrow|\uparrow\rangle_{S}
\end{aligned}
$$

\footnotetext{
${ }^{3}$ So Rovelli's project is incomplete in three ways: the principles fail to be motivated independently of the formalism, the postulates do not fall directly out of the first discussion, and the derivation lacks some rigor, especially with the ad hoc introduction of the principle that allows for superpositions. Despite Rovelli's failure to meet his own desiderata, his view does address familiar problems in an interestingly new way, and may have merits on its own that forgive this incompleteness.

${ }^{4}$ This is just the Wigner's friend case, without any reference to human agents or consciousness. $S$ is the system, $O$ is the friend, and $P$ is Wigner.
} 
Here we have what is referred to in orthodox expositions of quantum theory as the "collapse of the state / wavefunction" (von Neumann's Process I).

Now consider observer $P$ who treats both the observer and system from our previous discussion as the quantum mechanical system $S+O$. $P$ treats both the state of the system $S$ as well as the measuring device of $O$, which has the eigenstates $\mid$ ready $\rangle_{O},|u p\rangle_{O}$, and $\mid$ down $\rangle_{O}$ corresponding to its readout of 'ready', 'up', and 'down'. If $P$ has not performed a measurement at $t_{2}$, and instead only knows that there is an interaction, then just given the linearity of quantum mechanics, the state transition according to $P\left(|\psi\rangle_{S O}\right)$ will be:

$$
\begin{aligned}
t_{1} & \rightarrow t_{2} \\
|\operatorname{ready}\rangle_{O}\left(\alpha|\uparrow\rangle_{S}+\beta|\downarrow\rangle_{S}\right) & \left.\rightarrow \alpha|u p\rangle_{O}|\uparrow\rangle_{S}+\beta \mid \text { down }\right\rangle_{O}|\downarrow\rangle_{S}
\end{aligned}
$$

This process, unlike (1), proceeds entirely according to the linear dynamics (von Neumann's Process II).

From this discussion, Rovelli derives the following principle (what he calls the "main observation"):

R1 "In quantum mechanics different observers may give different accounts of the same sequence of events." [Rov96, p. 4 $]^{5}$

The sequence of events from $t_{1}$ to $t_{2}$ are described quite differently from the perspective of $O$ and $P$. Particularly, $O$ reports an event at $t_{2}$ where there is a measurement of a determinate property, while $P$ reports an interaction between two quantum systems without any determinacy in the property of either subsystem $(O$ or $S)$.

Rovelli takes this principle and then analyzes various interpretations of quantum mechanics as attempts to weasel out of $\mathbf{R} \mathbf{1}$, that is, as attempts to say that (1) and (2) can't both be true. Collapse theories say that (1) but not (2) is true, because interaction with the macroscopic measuring device (or something) causes the collapse of $S$ 's state. Versions of Everett's theory say that (2) but not (1) is true, because wavefunctions never collapse. If the formalism really implies (1) and (2), all such theories constitute a denial of the validity of the quantum formalism in some circumstances. Other

\footnotetext{
${ }^{5}$ As pointed out to me in comments on an earlier draft of this paper, this principle bears some resemblance to Bohr's principle of complementarity, which should be carefully distinguished from the "orthodox" interpretation.
} 
interpretations, according to Rovelli, deny the basic formalism in some other way. These discussions are a little more complex, and their details need not concern is here. The discussion of collapse and Everettian theories will serve to see the general point.

Rovelli counters with a quite strong realism about the ordinary formulation of quantum theory, described by the following principle (with he calls "Hypothesis 2" or "Completeness"):

R2 "Quantum mechanics provides a complete and self-consistent scheme of description of the physical world, appropriate to our present level of experimental observations." [Rov96, p. 7]

Where quantum mechanics, for Rovelli, is just the formalism as it is normally used in basic applications. ${ }^{6}$ The conjunction of $\mathbf{R} \mathbf{1}$ and $\mathbf{R 2}$, understood in conjunction with the rest of the discussion, leads us to the following conclusion:

Quantum mechanics is a theory about the physical description of physical systems relative to other systems, and this is a complete description of the world. [Rov96, p. 7]

This is sensible enough; after all, it would seem that the only way for different observers to give different but correct accounts would be to make the truth of accounts relative to different observers. So, we must include information about the system/metasystem cut when we specifies states, observables, and quantities. That is, we must introduce a new indexical into the formalism: we speak of $|\psi\rangle_{S / O}$ and $|\psi\rangle_{S O / P}$, not $|\psi\rangle_{S}$ and $|\psi\rangle_{S O}$ simpliciter. At $t_{2}$ in the example above, we consider the following state ascriptions:

$$
\begin{aligned}
|\psi\rangle_{S / O} & =|\uparrow\rangle_{S} \\
|\psi\rangle_{S O / P} & \left.=\alpha|u p\rangle_{O}|\uparrow\rangle_{S}+\beta \mid \text { down }\right\rangle_{O}|\downarrow\rangle_{S}
\end{aligned}
$$

\footnotetext{
${ }^{6}$ It is crucial to note the significance of the formalism as it is normally used. In particular, you will not see ordinary physics textbooks discussing the wave function of the Entire Universe, nor will such a wave function play a role in any familiar application of the formalism. It is an entity that is quite familiar to philosophers of physics, but one that Rovelli would see as entirely outside the normal usage. The formalism as normally used relies on a system/observer split, where only the system (in some sense) is described in the formalism. As we will see later on, this understanding of quantum mechanics is crucial to understanding Rovelli's position.
} 
From this conclusion it quickly follows that "the notion of observer-independent description of the world" is inappropriate. Giving up this notion (like giving up the notion of absolute simultaneity in the case of special relativity) is supposed to put our worries about quantum mechanics at ease.

\section{Barrett on Relational Interpretations}

Rather than discussing Rovelli's view explicitly, Jeffrey Barrett [Bar99] assimilates Rovelli's interpretation to David Mermin's view, which he discusses in detail. According to Barrett, Mermin tries to "understand quantum mechanics in terms of statistical correlations without there being any determinate correlata that the statistical correlations characterize"[Bar99, p. 217]. Expanding further, he says that on Mermin's view,

physics, properly understood, is about correlations and only correlations. It is not about correlations between determinate physical records nor is it about correlations between any other determinate physical properties. Rather, physics is about correlations without correlata. According to Mermin, 'Correlations have physical realty; that which they correlate does not'7 [Bar99, p. 217].

To put it another way, just as we normally index quantum states to times, views like Mermin's introduce an additional index into the variables of the theory that refers to the branch of the wave function relative to which the variable has a certain value. In the cases above, $O$ would say that $S$ is spinup relative to $S$ being $|\uparrow\rangle_{S}$, and $S$ would be $|\uparrow\rangle_{S}$ relative to $O$ observing it to be so, while $O$ measures $S$ as spin-down relative to $S$ being $|\downarrow\rangle_{S}$, and $S$ is $|\downarrow\rangle_{S}$ relative to $O$ observing it to be so. All that we can say "absolutely" is that $S$ and $O$ are correlated in a certain way; any further statements are relative to choice of a certain branch.

Barrett makes three major arguments against Mermin's account [Bar99, pp. 218-9]: (i) relations are necessarily relations between determinate relata, that is, a relations-without-relata view is (either metaphysically or analytically) incoherent; (ii) on Mermin's interpretation, there are no determinate physical records, and thus there are no determinate mental records (if mental states supervene on physical states in the ordinary way), and thus there are

\footnotetext{
${ }^{7}$ Barrett quotes [Mer97, p. 2]
} 
no determinate experiences, and thus the theory makes no determinate empirical predictions; (iii) Mermin's version of the theory does not even predict the right correlations since it does not tell us how to update the quantum state after a measurement. These criticisms are quite damning, if accurate. It is not my purpose to say whether Barrett gives a fair account of Mermin's view, nor to give responses on behalf of Mermin. Instead, I will examine how Rovelli's account differs from the Mermin's view (as presented by Barrett), and ask whether Barrett's criticisms apply to Rovelli's account as well.

It turns out that there are a number of differences between Rovelli's view and the view that Barrett attacks. The most important thing to notice is that Rovelli indexes facts not to branches, but to a certain cut. Rovelli's account admits a distinction between types of relations: on the one hand, there are system-system relations, and, on the other, there are systemobserver relations. System-system relations are interactions among elements of the system that can become entangled quantum-mechanical correlations. System-observer relations are interactions between the system and observer such that a property of the system becomes actualized for the observer. While there is always in principle an observer who can treat any systemobserver relation as a system-system relation (e.g., moving from $O$ to $P$ ), that new observer will have different information about the system than the first, including different information about the determinacy of properties of the system. To make it sound less epistemic, the new observer will define a different cut between system and observer, and so the states, properties, etc., will, in general, be different.

From this, we can see that Rovelli would say something different about determinacy and relata/correlata. Insofar as we are looking for absolutely determinate relata, then Rovelli would agree that there are no such things. Rovelli's relations do hold between relata (different physical systems), and those relata can be determinate relative to system-observer relations. Determinacy is not entirely absent; it is just relativized to certain observer-system cuts. Furthermore, by performing the proper measurements (that is, by interacting with the system in the right ways), the observer can find determinate answers to any well-formed question (that is, the observer can render any particular property of the system determinate). What is more, different observers can get together and compare notes about their determinate results and they will find that they agree (though more on this later).

We can understand the peculiar relativity of determinacy better by asking why the unitary dynamics fails to apply in the system-observer interactions. 
This happens because of the incompleteness of $O$ 's description of the interaction. As Rovelli puts it:

From the point of view of $[P]$, the measurement is therefore a fully unitary evolution, which is determined by the interaction Hamiltonian between $O$ and $S$. An interaction is a measurement that brings the states to a correlated configuration. On the other hand, $O$ gives a dynamical desciption of $S$ alone. Therefore he can only use the $S$ Hamiltonian. Since between times $t_{1}$ and $t_{2}$ the evolution of $S$ is affected by its interaction with $O$, the description of the unitary evolution of $S$ give by $O$ breaks down. The unitary evolution does not break down for mysterious physical quantum jumps, due to unknown effects, but simply because $O$ is not giving a full dynamical description of the interaction. $O$ cannot have a full description of the interaction of $S$ with himself $(O)$ because his information is correlation, and there is no meaning in being correlated with oneself. [Rov97, p. 205]

We can make a useful analogy to thermodynamics. So long as I am dealing with a closed system, the second law of thermodynamics applies. Once I open the system up to an influx of energy from outside, the second law breaks down, but that is only because I am not giving a full description of the interaction. I can bring the energy source into my description of the system, and it "becomes" a closed system again, to which the second law applies perfectly well. Just as the order in an open system can increase, even though a closed-system description would not allow this, a non-dynamical description on a quantum system (i.e., one in which a measurement, and thus an intervention, has been made) can lead to a determinate result, even though a dynamical description (i.e., one that includes both system and measuring device) would not describe a determinate result. ${ }^{8}$

We can now provide answers to Barrett on Rovelli's behalf. First, counter to (i), it is not the case that relations necessarily depend on determinate relata. Models of relations with relata of a nondeterminate nature date back at

\footnotetext{
${ }^{8}$ This analogy is not completely felicitous, because one can justly call the closed system in thermodynamics the complete description, and treat the effects in the open-system description as merely artifacts of an incomplete description. This option is not available in the quantum case, which will be described below.
} 
least to Plato's Theaetetus, if not further. ${ }^{9}$ Similarly, it seems that if we look at the perfect symmetry in defining volumes by integrating over points, or defining points as by series of smaller and smaller volumes, or the symmetry between defining graph-structures by listing their edges or their vertices, and other such symmetries ${ }^{10}$, it appears that there is no more problem giving primary reality to relations and abstracting relata from them, than there is giving primary reality to relata and showing how relations supervene on them. Or consider the following possibility: we have discovered that things can be broken down into molecules, molecules into atoms, atoms into subatomic particles, those particles into more fundamental particles. One might suppose it likely that this process of discovering smaller-and-smaller constituents will bottom out somewhere (and some believe that we have already arrived at this level, even if we haven't discovered all its members). But it is also imaginable that we find sub-quark particles, and sub-sub-quark particles, and... so on and so on with no end. ${ }^{11}$ It would certainly be a strange world, but nothing seems obviously incoherent or inconceivable about it. In such a case, we would have compounds without (non-compound) constituents, a special case of relations without relata. ${ }^{12}$ Whether such theories have their difficulties, it is not the case that they can be dismissed summarily, without looking to the specifics of the view.

Whether relations-without-relata views hold up is itself probably beside the point for Rovelli, because, counter to (ii), determinate physical records do exist (relative to a cut), as do determinate experiences. Attempting to understand whether this determinacy is enough will occupy the remainder of this essay, as it still may have a sort of determinacy problem. But one should note that this view is crucially different from Mermin's view, because relations are not the only thing that has physical reality. In addition to relations, there are interventions that produce determinate physical records. Now, these records are themselves relational in the sense that their determinacy is relative to a cut, but this relationality is different than Mermin's

\footnotetext{
${ }^{9}$ Plato describes the Protagorean/Heraclitean theory of perception as being one built on relations between nondeterminate relata (so-called "pure flux").

${ }^{10}$ Perhaps including the symmetry between Humeanism (See David Lewis) and Causal Structuralism (See [Haw01]).

${ }^{11}$ This is just Paul Churchland's "onion-world" from [Chu85].

${ }^{12}$ That is, relations without non-relational relata. But it seems obvious that no relationalist could insist that there were no relata, just that there are no relata that aren't themselves relations.
} 
correlations (as seen above), and the description that focuses on the correlations (i.e., the one that treats the elements in question under the dynamics) is no more fundamental than the other (and in fact, a description that was entirely at the level of the dynamics would never get any information about determinate properties).

Counter to (iii), Rovelli (unlike Mermin) can tell each observer how to update his states after a measurement: just as the standard formalism tells us to. It is important here to qualify the point made earlier: Rovelli, perhaps incautiously, says that $O$ does not give "a full dynamical description of the interaction" of $O$ with $S$. One could read this with emphasis on the description not being full. This leads to the question: how could an incomplete description solve the problem of metaphysical determinacy. So it would seem that Rovelli didn't have a good answer to (ii) after all. Furthermore, one would also be lead to ask: why would anyone ever be justified in using an incomplete description if one knows (or knows that there could be) a more complete description? ${ }^{13}$ I suggest that if this move is going to do what Rovelli thinks it does, we must instead read the point at issue with emphasis on the description not being fully dynamical. Here, we have two different types of description: ${ }^{14}$ partially dynamical description plus non-dynamical intervention, and fully dynamical. We haven't got a distinction between more and less complete descriptions of the same thing, because, first, the two descriptions are different, relying on different information, but neither is more complete than the other, second, $O$ can gather more information than $P$ at certain points in the process (like the determinate state of $S$ at $t_{2}$ ), and, third, because they aren't descriptions of the same thing in the sense of the same state, since states are individuated relative to cuts, not independent of them (though, of course, it is the same system). The state of $S$ relative to $S / O$ is not identical to the state of $S$ relative to $S O / P$.

\footnotetext{
${ }^{13}$ Thanks to Jeff Barrett and Craig Callender for pointing out the problems with this reading.

${ }^{14}$ Even this is not quite right. The first point is not even a description, exactly, because quantum mechanics cannot describe what will happen in an intervention, only that there is some probability of certain things happening.
} 


\section{A Puzzle About Relative States}

We can recapture a similar worry about relational quantum mechanics by comparing what happens at $t_{2}$ for $O$ to what happens at the time of $P$ 's measurement, $t_{3}$. As noted by von Neumann [vNe32], no matter where one places a cut, the probabilities will always agree, so, prior to the actual measurements, $O$ and $P$ will agree on the probabilities of finding states associated with the electron's being spin-up. According to the Malus-Born law, at $t_{2}$, there is a probability of $\left|\alpha^{2}\right|$ that $O$ will find $S$ to be x-spin-up and a probability of $\left|\beta^{2}\right|$ that $O$ will find it down. Now suppose that $O$ measures 'up'. So, according to the relational interpreation, the state of $S$ for $O$ is actually $|\psi\rangle_{S / O}=|\uparrow\rangle_{S}$. Further, according to the relational interpreation, the state of $S+O$ for $P$ is $|\psi\rangle_{S O / P}=\alpha|u p\rangle_{O}|\uparrow\rangle_{S}+\beta \mid$ down $\rangle_{O}|\downarrow\rangle_{S}$. According to the Malus-Born law, the probabilty that $P$ will find the state at $t_{3}$ to be $|u p\rangle_{O}|\uparrow\rangle_{S}$ (electron spin-up and $O$ indicating 'up') is $\left|\alpha^{2}\right|$, and the probability of $\mid$ down $\rangle_{O}|\downarrow\rangle_{S}$ is $\left|\beta^{2}\right|$. So, as von Neumann taught us, the probabilities agree. But notice: if we are to take $\mathbf{R} 2$ seriously, nothing said so far prevents it from being the case that $P$ finds $\mid$ down $\rangle_{O}|\downarrow\rangle_{S}$ at $t_{3}$, and thus $S$ being spin-down for $P$, even though $S$ was spin-up for $O$ !

Things get even more interesting. $P$ will never know that $S$ was spin-up for $O$, because, for $P, S$ was spin-down for $O$. That is, $P$ measures not $S$ 's state in isolation $\left(|\psi\rangle_{S / P}\right)$, but $S+O$. And, for $P$, $O$ 's state is 'down.' So, for $P, O$ 's observation is consistent with $P$ 's own observation of $S$ (as part of the $S+O$ system). So, while $O$ observed 'up' accord to $O, O$ observed 'down' according to $P$. Any any subsequent observations $P$ does are going to be consistent with all the other observations that $P$ has done. But here's the puzzle: we have parallel sets of consistent events relative to $O$ and $P$, which nevertheless disagree. Consider a case where $O$ is Schrödinger's cat and $P$ is the evil experimenter. The cat could be dead for the cat, while he's alive for $P$. If you think that there is a problem with spontaneous reanimation, then you might think there is a problem with the relational interpretation.

You might think that one could solve the "dispute" between $P$ and $O$ at $t_{2}$ over whether the state of $S$ has collapsed, at least in principle. After all, we know we can specify an observable that will distinguish between $S$ being in a superposition and $S$ having collapsed without doing anything that would actually collapse $S$ or reveal its state. Indeed, as Wigner has shown, it is possible to do so, and so it might be possible to adjudicate the dispute. This too fails, however, because the observable must be specified relative to a 
cut. It is not the case that there is some $\hat{A}$ that each observer could apply to $|\psi\rangle_{S}$. Instead, there is a $\hat{A}_{S / O}$ that $O$ can apply to $|\psi\rangle_{S / O}$ and a $\hat{A}_{S O / P}$ that $O$ can apply to $|\psi\rangle_{S O / P}$. Now, according to Rovelli, the former observable at (or just after) $t_{2}$ will indicate that the system is not in a superposition, while the later will indicate that it is. This renders no contradiction, as both the observables and the states they get applied to are different.

One might put the point in a somewhat different way. ${ }^{15}$ There is actually some $\hat{A}$ that each observer could apply to to $S$ to determine how the collapse went, whether the cat collapsed it or the scientist did. But if one could measure $\hat{A}$, the description and the result would be relative to some enormous measuring apparatus (if such was even possible). Say that $\hat{A}$ tells you that $S$ was in a superposition at $t_{2}$. This is entirely compatible with $S$ being in a determinate state at $t_{2}$ relative to $O$.

So we won't solve the puzzle this way. In other words, the puzzle is not a violation of anything observable.

\section{Canonical Cuts}

One way to solve the problem might be to suppose that there are canonical ways to cut up particular physical situations. In all the contexts we're interested in, it will be obvious where to place the cut between system and metasystem. This response shares affinities with Bohr's interpretation, which insists on careful specification of the measuring apparatus and the system in a way that lends itself to an unambiguous interpretation and communication of the measurement.

If I'm interested in testing some predictions about superconducting nanobelts, I will go down to the lab down in the basement of the physics building, get out my sample, position it among various standard bits of equipment, and start running some tests. It is obvious what the system whose states or properties I'm interested in is: the sample of nanobelts. It is equally obvious what counts as part of the measurement system: the various bits of lab equipment that I use to perform the experiment. One could argue that this will be the case for any physical situation of interest, and thus that there are canonical observer-system cuts. Furthermore, canonical cuts may be helpful in explaining experience, if we can explain how such cuts match up with our ordinary perspectives.

\footnotetext{
${ }^{15}$ I'm indebted to Craig Callender for this alternative.
} 
There is something dissatisfying, and perhaps even ad hoc about this solution. Must we really introduce such canonical cuts? Are there really canonical cuts in all physical situations of interest, or merely in a narrow class of circumscribed experimental situations? On the very frontiers of science, experimentalists must work very hard to design measuring equipment that can actually isolate interesting phenomena. It is very far from obvious how to separate system from observer. What happens if we insist that any old cut will do?

\section{Is Quantum Consistency Enough?}

Without adverting to something like a canonical cut, the relationalist has a ready answer to this paradox: the problem only gets off the ground if one assumes some absolute standpoint from which to view the situation. Because of the self-consistency of the quantum framework, any possible standpoint is going to predict consistent results among any subsystems of the system under consideration. This consistency is taken as a cardinal virtue of the relational interpretation by its adherents:

This internal self-consistency of the quantum formalism is general, and it is perhaps its most remarkable aspect. This self consistency is taken in relational quantum mechanics as a strong indication of the relational nature of the world. [Laud05]

Just as a number of seeming paradoxes in relativity disappear when we let go of the notion of absolute simultaneity, this paradox disappears when we let go of the absolute standpoint, of absolute states, quantities, and events. Once we give up the idea of absolute state, we are unable to state the paradox; any description allowable by quantum mechanics will allow full consistency. We can describe the match between the $S$ and $O$ from the point of view of $P$, and we can compare the points-of-view of $O$ and $P$ from yet another observer, $Q$, and in all of these situations, the results will come out consistent. It is only if we assume that different observers are embedded in a larger, non-quantum view-from-nowhere that paradoxes arise. As Rovelli says,

the notion "A system $O$ has information about a system $S$ " is a physical notion that can be studied experimentally (by a third observer), in the same way as any other physical property of a 
system. In particular, the question "Do observers $O$ and $[P]$ get the same answers out of a system $S$ ?" is a meaningless question. It is a question about the absolute state of $O$ and $[P]$. What is meaningful is to reformulate this question in terms of some observer. [Rov97, p. 204]

That $O$ observed 'spin-up' is not a fact that can be stated absolutely. We cannot ask a statement about $O$ 's knowledge without reference to another observer. From $t_{2}$ to $t_{3}$, we can consider questions about the answer that $O$ gets from the standpoint of $O$, who will arrive at a determinate answer because of the break down of the unitary dynamics, and we can consider from the standpoint of $P$, where $O$ will at first be correlated with the superposed states of $S$, and then become determinate when the dynamics breaks down because of $P$ 's own intervention.

The move here can again be understood by analogy, this time to special relativity (so, we end where we begin). In special relativity, certain paradoxes arise, where observer $Q$ observes event $A$ to precede event $B$, while observer $R$ sees $A$ following $B$. The contradiction disappears when we give up the notion of absolute simultaneity. Likewise, the contradictions disappear in quantum mechanics when we give up the notion of absolute state or absolute physical quantities. In special relativity, certain invariants remain, like the worldlines of particles through spacetime. Likewise, in relational quantum mechanics, the physical relations between systems remain invariant. ( $O$ and $P$ agree about the relation of $O$ to $S$, but at $t_{2}$ they disagree about the determinacy of each.)

The view has its attractions. Unlike realistic-collapse theories, it takes the predictions of the formalism with full seriousness. It will in fact conflict with collapse theories when it comes to whether $P$ could measure a superposition $(\hat{A})$ at $t_{2}$. Unlike ordinary no-collapse theories, it gives us determinate measurement records (as determinate as we could want) without adverting to a dualistic solution to do so, for the theory posits no extra entities beyond what the ordinary formalism requires, nor does it require any minds or worlds or suchlike. The theory doesn't even require reference to consciousness or any human agents whatsoever. The processes of measurement considered are mundane old physical interactions, ones that can (from a certain pointof-view) be described quantum-mechanically. On the other hand, we fail to capture the security of the unified world of classical mechanics. Maybe the price isn't so much to pay, given the dismal array of alternative options. 


\section{References}

[Bar99] Barrett, J.A., The Quantum Mechanics of Minds and Worlds, (Oxford: Oxford UP, 1999).

[Chu85] Churchland, P.M. (1985), "Conceptual Progress and Word/World Relations: In Search of the Essence of Natural Kinds," Canadian Journal of Philosophy 15: 1-17.

[Fink96] Finkelsten, D.R., Quantum Relativity: A Synthesis of the Ideas of Einstein and Heisenberg, (Berlin: Springer-Verlag, 1996).

[Fink04] Finkelstein, D.R. (2004) "Finite Quantum Relativity," Unpublished manuscript, Revised 10-11-2004, Retrieved from $<$ http://www.physics.gatech.edu/people/faculty/finkelstein/ FQR02.pdf $>$ on 7-20-2005.

[Haw01] Hawthorne J., "Causal Structuralism," Noûs, Volume 35, Supplement 1, October 2001, pp. 361-378(18).

[Laud05] Laudisa, F. \& Rovelli, C., "Relational Quantum Mechanics", The Stanford Encyclopedia of Philosophy (Fall 2005 Edition), E. N. Zalta (ed.), http://plato.stanford.edu/archives/fall2005/entries/ qm-relational/

[Mer97] Mermin, N.D. (1997), "What is Quantum Mechanics Trying to Tell Us?" LANL archive preprint.

[Rov96] Rovelli, C., "Relational Quantum Mechanics," Int. J. of Theor. Phys. 35 (1996) pp. 1637-78, Revised: arXiv:quant-ph/9609002 v2 $24 \mathrm{Feb} 1997$.

[Rov97] Rovelli, C., "Halfway Through the Woods: Contemporary Research on Space and Time," The Cosmos of Science: Essays of Exploration, ed. J. Earman \& J.D. Norton, (Pittsburgh: U Pitt Press: 1997), pp. 180-223.

[vNe32] von Neumann, J. (1932), Mathematische Grundlagen der Quantenmechanik (Julius Springer-Verlag, Berlin, 1932). 